\title{
Hydrogen saline suppresses neuronal cell apoptosis and inhibits the p38 mitogen-activated protein kinase-caspase- 3 signaling pathway following cerebral ischemia-reperfusion injury
}

\author{
DA LI and YANQIU AI \\ Department of Anesthesiology, First Affiliated Hospital of Zhengzhou University, Zhengzhou, Henan 450000, P.R. China
}

Received July 13, 2016; Accepted May 2, 2017

DOI: $10.3892 / \mathrm{mmr} .2017 .7294$

\begin{abstract}
Cerebral ischemia-reperfusion injury (CIRI) is a serious pathological disease that is associated with a high rate death and disability. Saturated hydrogen $\left(\mathrm{H}_{2}\right)$ saline exhibits brain protective functions through anti-inflammatory, antioxidant and antiapoptotic effects. The present study investigated the potential treatment effects of $\mathrm{H}_{2}$ on CIRI. In addition, the potential protective mechanisms of $\mathrm{H}_{2}$ in the prevention of CIRI were investigated. Adult, male Sprague-Dawley rats $(n=60)$ were randomly divided into the following three groups: Sham-operated group; IR group; and IR $+\mathrm{H}_{2}$ group (0.6 mmol/1, $0.5 \mathrm{ml} / \mathrm{kg} /$ day). Hematoxylin and eosin, and TUNEL staining were performed for histopathological analysis and investigation of apoptosis, respectively. In addition, the protein expression of caspase-3, p38 mitogen-activated protein kinase (MAPK) and phosphorylated-p38 MAPK in the cortex were measured by western blotting analysis. These results demonstrated that $\mathrm{H}_{2}$ significantly reduced the number of apoptotic cells, and the protein expression of p38 MAPK and caspase-3, compared with the IR group. These effects may be associated with the p38MAPK signaling pathway.
\end{abstract}

\section{Introduction}

Cerebral ischemia-reperfusion injury (CIRI) causes serious complications and permanent disability. The rapid recovery of blood flow and supply of oxygen causes the release of inflammatory cytokines, which subsequently causes brain injury (1). The mechanisms associated with CIRI include inflammation, oxidative stress and apoptosis. Although multiple mechanisms contribute to the pathology, the importance of apoptosis in the pathology of CIRI has been previously reported (2).

A previous study demonstrated that $\mathrm{H}_{2}$ selectively reduces the number of inflammatory cells and levels of reactive oxygen

Correspondence to: Professor Yanqiu Ai, Department of Anesthesiology, First Affiliated Hospital of Zhengzhou University, 1 East-Jianshe Road, Zhengzhou, Henan 450000, P.R. China

E-mail: aiyanqiu82@163.com

Key words: hydrogen rich saline, apoptosis, p38 mitogen-activated protein kinase, caspase-3, ischemia-reperfusion injury species (ROS), which reduces apoptosis (3). Saturated $\mathrm{H}_{2}$ saline has been demonstrated to exhibit various biological properties, including anti-inflammatory and antioxidant effects (4). Previous reports have demonstrated that $\mathrm{H}_{2}$ saline exerts neuroprotective effects in IRI models $(5,6)$. The neuroprotective effects of $\mathrm{H}_{2}$ may be associated with its antioxidant and antiapoptotic properties. In addition, it was previously demonstrated that the neuroprotective effects of $\mathrm{H}_{2}$ during ischemia injury were primarily via antiapoptosis effects (7). Nevertheless, the mechanisms underlying the antiapoptotic effect of $\mathrm{H}_{2}$ saline remain unclear.

p38 mitogen-activated protein kinase (MAPK) is one of the MAPK subfamilies that regulate cell growth, differentiation and stress responses (8). The activation of p38 MAPK leads to the stimulation of apoptosis, which is supported by a report that administration of SB203580, a p38 MAPK inhibitor, decreased neuronal apoptosis by inactivating ROS (9). Activated p38 MAPK leads to neuronal death primarily through alterations in the expression of proteins involved in apoptosis, including caspase-9, caspase-3 and the apoptosis regulator Bcl-2/apoptosis regulator BAX. A previous report confirmed that the $\mathrm{p} 38$ MAPK signaling pathway activates the proapoptotic protein caspase-3 (10) to induce apoptosis in ischemic disease (11).

Therefore, the present study employed saturated $\mathrm{H}_{2}$ saline in a CIRI model, and aimed to investigate whether saturated $\mathrm{H}_{2}$ attenuates neuronal apoptosis via the p38 MAPK-caspase-3 signaling pathway.

\section{Materials and methods}

Reagents. Saturated $\mathrm{H}_{2}$ saline was provided by the Department of Diving Medicine, Faculty of Navy Medicine, Second Military Medical University (Shanghai, China). Rabbit polyclonal antibodies against caspase-3 (1:600, ab52293) were purchased from Abcam (Cambridge, UK), p38 (BM2044) was provided by BIOSS (Beijing, China). The immunohistochemistry diaminobenzidine reagent (AR1024) was purchased from Wuhan Boster Biological Technology Ltd. (Wuhan, China) and the TUNEL kit (KGA7026-52) was purchased from Nanjing KeyGen Biotech Co., Ltd. (Nanjing, China).

Animals. Male Sprague-Dawley rats ( $\mathrm{n}=60$; age, 80-100 days; body weight, 220-250 g) were provided by the Experimental Animal Center of the First Affiliated Hospital of Zhengzhou 
University (Zhengzhou, China). Rats were housed in individual cages at $22-24^{\circ} \mathrm{C}$ with controlled humidity and a $12-\mathrm{h}$ light/dark cycle. The rats had free access to food and water. Rats were randomly assigned into the following three treatment groups: Control group $(n=20)$; IR group $(n=20)$; and $\mathrm{IR}+\mathrm{H}_{2}$ (IRH) group $(\mathrm{n}=20)$. Briefly, mice were anesthetized by facemask with $3 \%$ enflurane. A nylon filament with a rounded silicone $0.19-\mathrm{mm}$ diameter tip was inserted into the right common carotid artery to induce ischaemia and removed 60 min later. Local cerebral blood flow was observed by transcranial laser Doppler (PeriFlux System 5000; Perimed AB, Järfälla, Sweden) to confirm the occlusion. Body temperature was monitored during surgery with a rectal probe and maintained at $37.0 \pm 0.5^{\circ} \mathrm{C}$ using a heating lamp.

Rats in the IRH group were given $0.5 \mathrm{ml} / \mathrm{kg} /$ day saturated $\mathrm{H}_{2}$ saline $(0.6 \mathrm{mmol} / \mathrm{l})$ intraperitoneally three days prior to CIRI and immediately following $24 \mathrm{~h}$ of reperfusion, for a total of two injections. Rats in the IR group received the same dose of normal saline instead. After $24 \mathrm{~h}$ of reperfusion, all animals were sacrificed and tissue samples were obtained for examination. All efforts were made to minimize the experimental error and the number of animals used. All animal experiments were approved by the Animal Ethics Committee of Zhengzhou University.

Hematoxylin and eosin $(H \& E)$ staining. After $24 \mathrm{~h}$ of reperfusion, the rats were anaesthetized with $3 \%$ enflurane and perfused intracardially with $0.9 \%$ saline, followed by $4 \%$ paraformaldehyde in PBS. Brains were removed quickly and transferred to $4 \%$ buffered paraformaldehyde at $4^{\circ} \mathrm{C}$ for $24 \mathrm{~h}$. The brain slices were subsequently paraffin embedded and cut into $4 \mu \mathrm{m}$ thick serial sections. The sections were then incubated overnight at $4^{\circ} \mathrm{C}$ in a moist chamber. Tissues were stained with $\mathrm{H} \& \mathrm{E}$ for histopathological analysis, and the staining was visualized by light microscopy with $\mathrm{x} 40$ objectives (Olympus Corporation, Tokyo, Japan). Slides were subsequently assessed in a blind manner by a pathologist for the following: Neutrophilic infiltrate; hemorrhage; lymphohistiocytic infiltrate; and cerebral cell apoptosis.

Evaluation of neurological deficit. Berderson's neurological examination scoring system (12) was used to evaluate animal behavior. Rats scored 0 if they had no obvious deficit (normal), 1 if they failed to fully extend the left forepaw (mild deficit), 2 if they circled towards the contralateral side (moderate deficit) and 3 if they lost righting reflexes and the ability to walk (severe deficit). The neurological deficit scores of each rat was evaluated carefully 6,12 and $24 \mathrm{~h}$ following reperfusion by an observer who was blinded to the study.

In situ apoptosis assay. Cell apoptosis was investigated using a TUNEL kit. Sections $(4 \mu \mathrm{m})$ were deparaffinized with xylene and rehydrated at $60^{\circ} \mathrm{C}$ with graded ethanol $(100,95,90,80$, $70 \%$ ). Sections were incubated with $20 \mu \mathrm{g} / \mathrm{ml}$ proteinase $\mathrm{K}$ working solution for $15 \mathrm{~min}$ at room temperature. Sections were washed three times with PBS and incubated with TUNEL reagent for $1 \mathrm{~h}$ at $37^{\circ} \mathrm{C}$. Sections were incubated with alkaline phosphatase for $1 \mathrm{~h}$ at $37^{\circ} \mathrm{C}$. Following washing with PBS three times, sections were stained with nitro blue tetrazolium chloride and 5-bromo-4-chloro-3-indolyl phosphate. A total of four fields were randomly examined at magnification x 20 . A total of 100 cells were counted in each field and the data are presented as the percentage of TUNEL-positive cells/total cells in each field.

Immunohistochemical results. The brain tissue was harvested after $24 \mathrm{~h}$ of reperfusion. Samples were fixed in $4 \%$ paraformaldehyde at $24^{\circ} \mathrm{C}$, embedded in paraffin and cut into $4 \mu \mathrm{m}$ slices. Sections were deparaffinized with xylene and rehydrated at $60^{\circ} \mathrm{C}$ with graded ethanol $(100,95,90,80,70 \%)$. Endogenous peroxidase activity was blocked using $3 \%$ hydrogen peroxide for $30 \mathrm{~min}$ at room temperature, followed by $5 \%$ normal goat serum (AR0009; Wuhan Boster Biological Technology Ltd.) for $1 \mathrm{~h}$ to block non-specific protein interactions. The sections were subsequently incubated overnight at $4^{\circ} \mathrm{C}$ with an anti-p38 MAPK antibody (1:100). The negative control group was incubated with PBS instead of the primary antibody. Following three washes with PBS, the slides were incubated with a biotinylated goat anti-mouse horseradish peroxidase conjugated secondary antibody (1:100, BA1051; Wuhan Boster Biological Technology Ltd.) for $2 \mathrm{~h}$ at room temperature. The sections were washed with PBS again, and incubated with the kit-provided horseradish peroxidase (HRP)-streptavidin for $30 \mathrm{~min}$ at room temperature. The peroxidase reaction was visualized using $0.05 \%$ diaminobenzidine $+0.01 \%$ hydrogen peroxide. Immunhistochemical procedures were performed in accordance with the manufacturer's protocols. The positive cells were visualized by a microscope at $x 400$ magnification. Fields $(\geq 10)$ were randomly selected for visualization. The positive cell index (PCI = apoptotic cells/total cells) was calculated and used to indicate p38MAPK expression levels.

Western blot analysis. Total protein was extracted from brain tissues using whole cell lysis buffer (WD2072; Beyotime Institute of Biotechnology, Shanghai, China). Protein concentrations were determined using an enhanced Bicinchoninic Acid Protein Assay kit (P10310; Beyotime Institute of Biotechnology). Protein samples were mixed with 5X SDS-PAGE sample loading buffer, denatured at $95-97^{\circ} \mathrm{C}$ for $5 \mathrm{~min}$ and stored at $-80^{\circ} \mathrm{C}$ until further analysis. Total proteins $(20 \mu \mathrm{g})$ were separated by $10 \%$ SDS-PAGE and transferred to polyvinylidene difluoride (PVDF) membranes. The PVDF membrane was blocked with $5 \% \mathrm{v} / \mathrm{v}$ nonfat milk in Tris-buffered saline $/ 0.1 \%$ Tween- 20 buffer and probed overnight at $4{ }^{\circ} \mathrm{C}$ with the following primary antibodies: p38MAPK (1:600), caspase-3 (1:600) or $\beta$-actin (1:600). The membranes were subsequently incubated with goat anti-rabbit HRP-conjugated secondary antibody (1:10,000; AB14387; Abcam) for $1 \mathrm{~h}$ with gentle agitation at room temperature. The membranes were incubated in enhanced chemiluminescence substrate (AR0163-1; Wuhan Boster Biological Technology Ltd.) reagent for $60 \mathrm{sec}$ and exposed to autoradiography film for visualization of the bands. Densitometric analysis of the bands was performed using Image J version 1.6.0 (National Institutes of Health, Bethesda, MD, USA). Relative optical densities of protein bands were calibrated against $\beta$-actin and normalized to the levels in the sham-operated rats.

Statistical analysis. Data are presented as the mean \pm standard error of the mean. Analysis was performed using SPSS 13.0 
software (SPSS, Inc., Chicago, IL, USA). One-way analysis of variance was used to compare differences between experimental groups, followed by Dunnetts post hoc test. $\mathrm{P}<0.05$ was considered to indicate a statistically significant difference.

\section{Results}

Histopathological examination by $H \& E$ staining. As demonstrated in Fig. 1, no neuronal damage or infiltrating inflammatory cells were visible in the sham group. Neurons were eumorphic with normal cellular architecture. The nuclei were in the cell center and clearly stained. In the IR group, ischemic tissue was characterized by a large number of degenerated and necrotic cells (black arrow), and inflammatory cells infiltration (yellow arrow). In the IRH group, there was moderate neuronal damage in the ischemic area (Fig. 1). These data indicate that $\mathrm{H}_{2}$ may reduce CIRI.

Neurological deficit scores. As presented in Table I, the behavior of the sham group indicated no neurological deficit. Rats in the IRH group exhibited increased neurological deficit compared with the sham group after $12 \mathrm{~h}$ reperfusion; however, after $24 \mathrm{~h}$ reperfusion the score in the IRH group was significantly reduced compared with the IR group, although this remained increased compared with the sham group. These data demonstrate that pretreatment with $\mathrm{H}_{2}$ saline may effectively reduce neurological deficits following CIRI.

Apoptotic cell analysis. As demonstrated in Fig. 2, the number of TUNEL-positive cells was significantly increased in the IR group compared with the sham group, and treatment with $\mathrm{H}_{2}$ saline led to a reduction in the number of apoptotic cells compared with the IR group.

Immunohistochemistry of p38 MAPK. In the sham group, few cells stained positively for p38 MAPK, and p38 MAPK expression was significantly increased in the IR group compared with the sham group. In the IRH group, the number of p38 MAPK stained cells was reduced compared with the IR group (Fig. 3).

$\mathrm{H}_{2}$-rich saline reduces IR-induced p38 MAPK and caspase- 3 overexpression. Western blot analysis demonstrated that the protein expression of p38 MAPK was significantly increased in the IR group compared with sham group (Fig. 4). However, treatment with $\mathrm{H}_{2}$ saline in the IRH group significantly reduced the expression of p38 MAPK compared with the IR group (Fig. 4). Similar effects were also observed for caspase-3 protein expression (Fig. 5).

\section{Discussion}

$\mathrm{H}_{2}$ saline has been used in various organ ischemic models, including myocardium, liver and cerebral models (13-15); however, the mechanism by which $\mathrm{H}_{2}$ saline protects against CIRI remains to be established. The results of the present study demonstrated that $\mathrm{H}_{2}$ ameliorated apoptotic cell death and inflammatory cell infiltration in the cerebral cortex of rats with CIRI.

There has been increased focus on the importance of apoptosis in the progression of CIRI $(16,17)$. In a normal physiological
Table I. Results of neurological evaluation in rats following 90 min cerebral artery occlusion and 6,12 or $24 \mathrm{~h}$ reperfusion.

\begin{tabular}{lccc}
\hline & \multicolumn{3}{c}{ Duration of reperfusion, $\mathrm{h}$} \\
\cline { 2 - 4 } Group & 6 & 12 & 24 \\
\hline Sham & $0.0 \pm 0.0$ & $0.0 \pm 0.0$ & $0.0 \pm 0.0$ \\
IR & $2.18 \pm 0.59^{\mathrm{a}}$ & $2.01 \pm 0.49^{\mathrm{a}}$ & $1.82 \pm 0.38^{\mathrm{a}}$ \\
IRH & $2.01 \pm 0.43^{\mathrm{a}}$ & $1.86 \pm 0.38^{\mathrm{a}}$ & $1.58 \pm 0.36^{\mathrm{a}, \mathrm{b}}$ \\
\hline
\end{tabular}

${ }^{\mathrm{a}} \mathrm{P}<0.05$ vs. sham; ${ }^{\mathrm{b}} \mathrm{P}<0.05$ vs. IR. $\mathrm{n}=6$. IR, ischemia-reperfusion; IRH, IR with saturated hydrogen saline treatment.
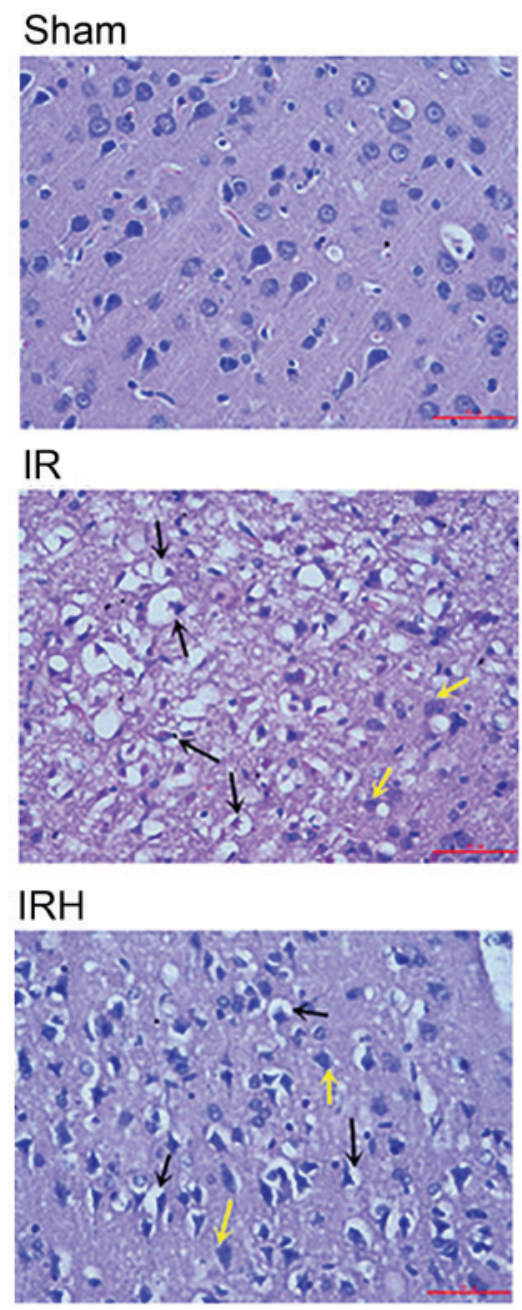

Figure 1. Histopathological examination by hematoxylin and eosin staining. Differences were observed in brain histology between the two experimental groups. Brain hemorrhage, edema, influx of inflammatory cells and fibrin deposition were observed in IR rats. By contrast, in the IRH group, the brain damage was alleviated, indicating that hydrogen saline may protect against cerebral IR injury. Bar, $50 \mu \mathrm{m}$. Black arrow, necrotic cells; yellow arrow, inflammatory cells infiltration. IR, ischemia-reperfusion; IRH, IR with saturated hydrogen saline treatment.

environment, a dynamic balance exists between apoptosis and the natural antiapoptotic defense system. An antiapoptotic effect of $\mathrm{H}_{2}$-rich saline was reported in a neonatal hypoxia-ischemic rat model (18). In order to investigate whether $\mathrm{H}_{2}$-rich saline 

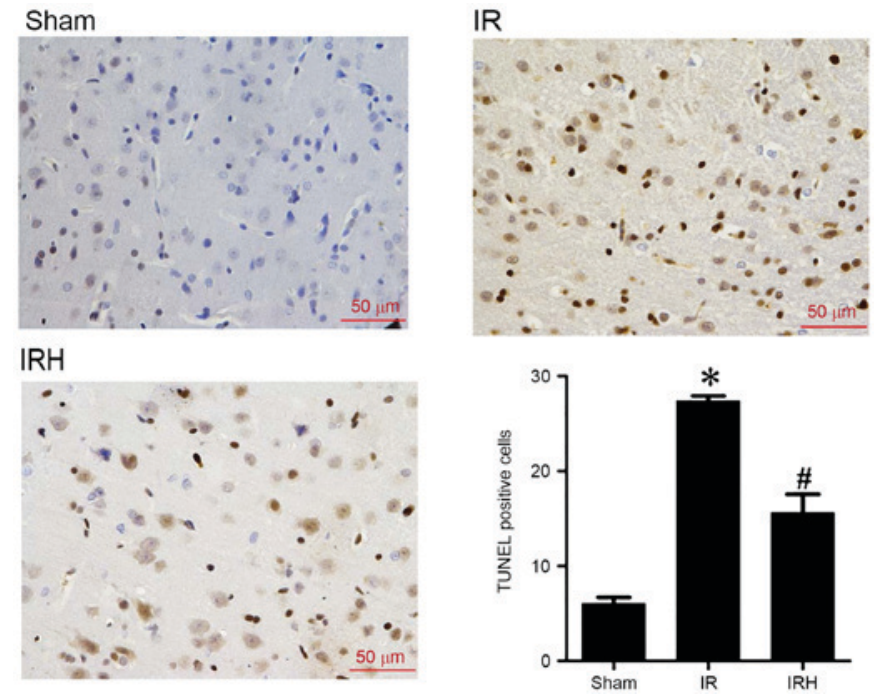

Figure 2. Effects of saturated hydrogen saline on cellular apoptosis. Rats in the IR group exhibited a significantly increased number of apoptotic (TUNEL-positive) cells compared with the sham group. Furthermore, the number of apoptotic cells in the IRH group was significantly reduced compared with the IR group. ${ }^{*} \mathrm{P}<0.05$ vs. sham; ${ }^{*} \mathrm{P}<0.05$ vs. IR. $\mathrm{n}=6$. IR, ischemia-reperfusion; IRH, IR with saturated hydrogen saline treatment.
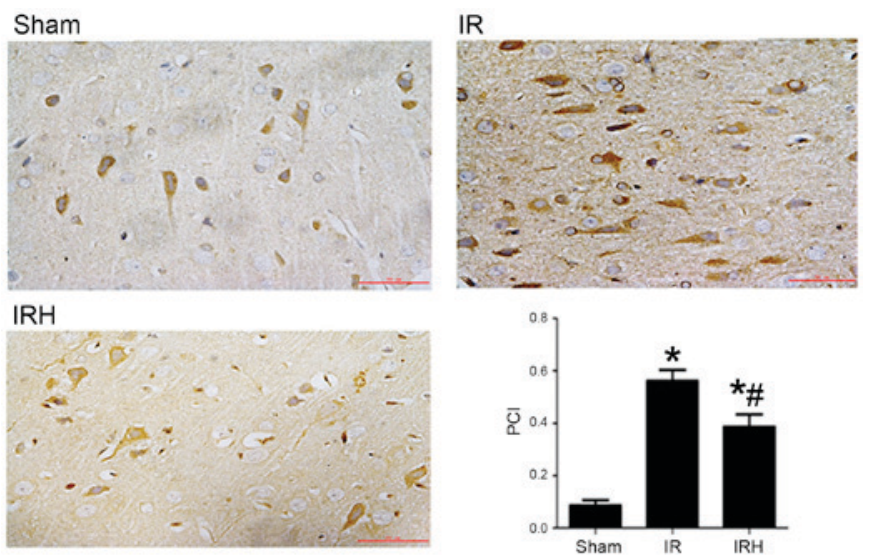

Figure 3. Immunohistochemical staining of p38 MAPK in sham, IR and IRH groups. Compared with the sham group, increased immunohistochemical reactivity for $\mathrm{p} 38$ MAPK was observed in the IR group. Treatment with hydrogen saline led to a reduction in the staining for p38 MAPK compared with IR group. ${ }^{*} \mathrm{P}<0.05$ vs. sham; ${ }^{\text {}} \mathrm{P}<0.05$ vs. IR. $\mathrm{n}=6$. MAPK, mitogen-activated protein kinase; IR, ischemia-reperfusion; IRH, IR with saturated hydrogen saline treatment.

exhibited an antiapoptotic effect in a rat model of CIRI, the present study examined the number of TUNEL positive cells and the protein expression of caspase-3, which is an important apoptosis protein. The number of apoptotic cells in the cortex was significantly increased in the IR group compared with the sham group. While in the IRH group, the number of apoptotic cells was significantly reduced compared with the IR group. These results demonstrated that $\mathrm{H}_{2}$-rich saline may have neuroprotective effects in CIRI, and this may primarily occur by inhibition of apoptosis.

p38 MAPK is one of a number of MAPKs in the MAPK signal transduction pathway, and has a role in cell proliferation, differentiation and cytokine synthesis $(19,20)$. In the process of CIRI, p38 MAPK is activated by the release of inflammatory
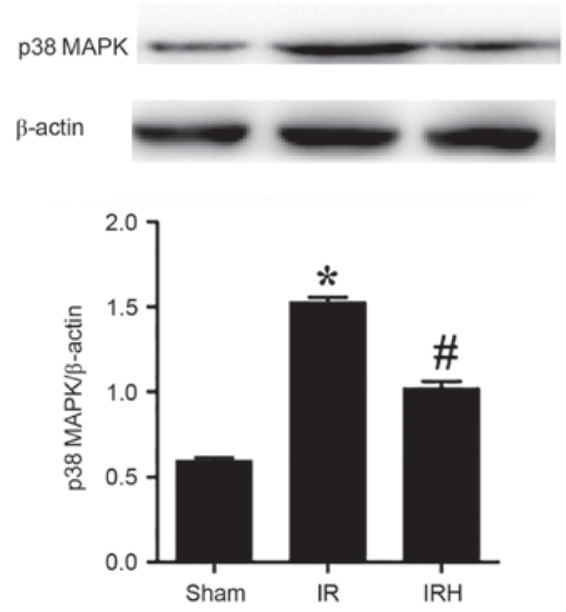

Figure 4. Expression of p38 MAPK protein in sham, IR and IRH groups. Representative western blots and quantified results for p38 MAPK protein expression in the rat cortex of sham, IR and IRH groups. As demonstrated, p38 MAPK protein expression was increased significantly in the IR group compared with the sham group. However, p38 MAPK expression was significantly reduced in the IRH treatment group compared with the IR group. Bars represent the mean \pm standard error of the mean. $n=6$. ${ }^{*} \mathrm{P}<0.05$ vs. sham; ${ }^{\#} \mathrm{P}<0.05$ vs. IR. MAPK, mitogen-activated protein kinase; IR, ischemia-reperfusion; IRH, IR with saturated hydrogen saline treatment.
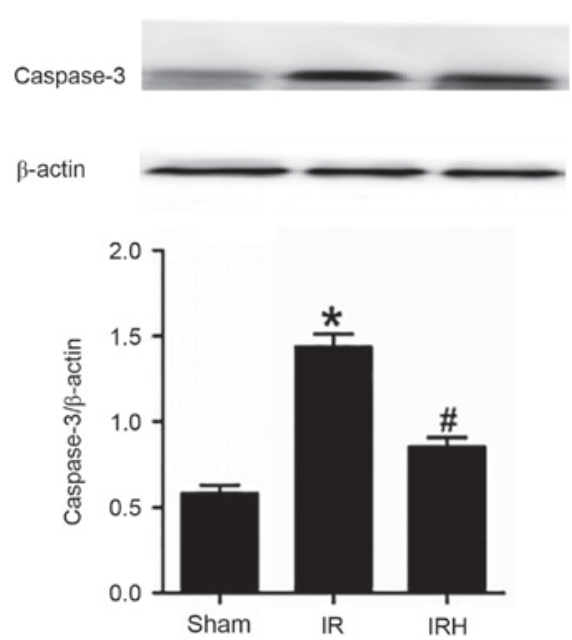

Figure 5. Expression of caspase-3 protein in sham, IR and IRH groups. Representative western blots and quantified results for caspase- 3 protein expression in the rat cortex of sham, IR and IRH groups. As demonstrated, caspase-3 protein expression was significantly increased in the IR group compared with the sham group. However, compared with the IR group, caspase-3 expression was significantly reduced in the IRH group. Bars represent the mean \pm standard error of the mean. $n=6$. ${ }^{*} \mathrm{P}<0.05$ vs. sham; ${ }^{*} \mathrm{P}<0.05$ vs. IR. IR, ischemia-reperfusion; IRH, IR with saturated hydrogen saline treatment.

cytokines or cell stress, and subsequently transferred into the nucleus. In the present study, immunohistochemical results indicated that there was a significant increase in the protein expression of p38 MAPK in the IR group compared with the sham group. In the IRH group, a significant decrease in the protein expression of p38 MAPK was observed compared with the IR group. Additionally, western blotting confirmed that treatment with $\mathrm{H}_{2}$-rich saline reduced the level of $\mathrm{p} 38$ MAPK protein compared with the IR group. Therefore, it was hypothesized that $\mathrm{H}_{2}$-rich saline may exhibit antiapoptotic 
neuroprotective effects via the p38 MAPK signaling pathway. However, the present study did not investigate the effect of a p38 MAPK signaling pathway inhibitor to confirm these results.

Caspase-3 is a member of the caspase family that includes caspases 1-12, and is an executor apoptotic protein in the apoptosis process (21). To elucidate the mechanisms by which $\mathrm{H}_{2}$ saline exerts antiapoptotic effects in neurons, the present study investigated the activation of caspase-3. The results demonstrated that CIRI promoted the expression of caspase-3, while in IRH group, levels of caspase-3 were reduced compared with the IR group. The present study demonstrated that $\mathrm{H}_{2}$-rich saline may protect against CIRI through antiapoptotic effects, potentially via reduced expression of caspase- 3 , which is an executor apoptotic protein.

In conclusion, $\mathrm{H}_{2}$-rich saline may reduce the expression of p38MAPK and inhibit neuronal apoptosis during CIRI. However, a limitation is that an inhibitor of p38MAPK was not used. The apoptotic signaling pathway is complex, and these results only demonstrate the role of caspase 3 in CIRI. In further studies we will attempt to investigate the role of caspase9, $\mathrm{Bcl} 2$ and Bax. Understanding the neuroprotective mechanisms induced by hydrogen during CIRI may aid in the identification of potential novel therapeutic targets.

\section{References}

1. Eltzschig HK and Eckle T: Ischemia and reperfusion-from mechanism to translation. Nat Med 17: 1391-1401, 2011.

2. Mattson MP, Duan W, Pedersen WA and Culmsee C: Neurodegenerative disorders and ischemia brain diseases. Apoptosis 6: 69-81, 2001.

3. Ohsawa I, Ishikawa M, Takahashi K, Watanabe M, Nishimaki K, Yamagata K, Katsura K, Katayama Y, Asoh S and Ohta S: Hydrogen acts as a therapeutic antioxidant by selectively reducing cytotoxic oxygen radicals. Nat Med 13: 688-694, 2007.

4. Xu XF and Zhang J: Saturated hydrogen saline attenuates endotoxin-induced acute liver dysfunction in rats. Physiol Res 62: 395-403, 2013

5. Liu Y, Liu W, Sun X, Li R, Sun Q, Cai J, Kang Z, Lv S, Zhang JH and Zhang W: Hydrogen saline offers neuroprotective by reducing oxidative stress in a focal cerebral ischemia-reperfusion rat model. Med Gas Res 1: 15, 2011.

6. Li J, Dong Y, Chen H, Han H, Yu Y, Wang G, Zeng Y and Xie K: Protective effects of hydrogen-rich saline in a rat model of permanent focal cerebral ischemia via reducing oxidative stress and inflammatory cytokines. Brain Res 1486: 103-111, 2012.

7. Cai J, Kang Z, Liu WW, Luo X, Qiang S, Zhang JH, Ohta S, Sun X, Xu W, Tao H and Li R: Hydrogen therapy reduces apoptosis in neonatal hypoxia-ischemia rat model. Neurosci Lett 441: $167-172,2008$
8. Arthur JS and Ley SC: Mitogen-activated protein kinases in innate immunity. Nat Rev Immunol 13: 679-692, 2013.

9. Ashraf MI, Ebner M, Wallner C, Haller M, Khalid S, Schwelberger H, Koziel K, Enthammer M, Hermann M, Sickinger S, et al: A p38MAPK/MK2 signaling pathway leading to redox stress, cell death and ischemia/reperfusion injury. Cell Commun Signal 12: 6, 2014.

10. Zhao Y and Wang S: Neural stem cell transplantation inhibits apoptosis through activation of MAPK/ERK signaling pathway in cerebral ischemia/reperfusion rats. Int J Clin Exp Neuro 1: $5-11,2013$

11. Su D, Ma J, Zhang Z, Tian Y and Shen B: Protective effects of UCF-101 on cerebral ischemia-reperfusion (CIR) is depended on the MAPK/p38/ERK signaling pathway. Cell Moll Neurobiol 36: 907-914, 2016.

12. Bederson JB, Pitts LH, Tsuji M, Nishimura MC, Davis RL and Bartkowski H: Rat middle cerebral artery occlusion: Evaluation of the model and development of a neurologic examination. Stroke 17: 472-476, 1986.

13. Fukuda K, Asoh S, Ishikawa M, Yamamoto Y, Ohsawa I and Ohta S: Inhalation of hydrogen gas suppresses hepatic injury caused by ischemia/reperfusion through reducing oxidative stress. Biochem Biophys Res Commun 361: 670-674, 2007.

14. Hayashida K, Sano M, Ohsawa I, Shinmura K, Tamaki K, Kimaru K, Endo J, Katayama T, Kawamura A, Kohsaka S, et al: Inhalation of hydrogen gas reduces infarct size in the rat model of myocardial ischemia-reperfusion injury. Biochem Biophys Res Commun 373: 30-35, 2008.

15. Ohsawa I, Nishimaki K, Yamagata K, Ishikawa M and Ohta S: Consumption of hydrogen water prevents atherosclerosis in apolipoprotein E knockout mice. Biochem Biophys Res Commun 377: 1195-1198, 2008.

16. Liu X, Zhao S, Liu F, Kang J, Xiao A, Li F, Zhang C, Yan F, Zhao H, Luo M, et al: Remote ischemic postconditioning alleviates cerebral ischemic injury by attenuating endoplasmic reticulum stress-mediated apoptosis. Transl Stroke Res 5: 692-700, 2014.

17. Wen XR, Tang M, Qi DS, Huang XJ, Liu HZ, Zhang F, Wu J, Wang YW, Zhang XB, Guo JQ, et al: Butylphthalide suppresses neuronal cells apoptosis and inhibits JNK-caspase 3 signaling pathway after brain ischemia/reperfusion in rats. Cell Mol Neurobiol 36: 1087-1095, 2016.

18. Cai J, Kang Z, Liu WW, Luo X, Qiang S, Zhang JH, Ohta S, Sun X, Xu W, Tao H and Li R: Hydrogen therapy reduces apoptosis in neonatal hypoxia-ischemia rat model. Neurosci Lett 441: $167-172,2008$.

19. Sucher R, Gehwolf P, Kaier T, Hermann M, Maglione M, Oberhuber R, Ratschiller T, Kuznetsov AV, Bösch F, Kozlov AV, et al: Intracellular signaling pathways control mitochondrial events associated with the development of ischemia/reperfusion-associated damage. Transpl Int 22: 922-930, 2009,

20. Jeong CW, Yoo KY, Lee SH, Jeong HJ, Lee CS and Kim SJ: Curcumin protects against regional myocardial ischemia/reperfusion injury through activation of RISK/GSK-3 $\beta$ and inhibition of p38 MAPK and JNK. J Cardiovasc Pharmocol Ther 17: 387-394, 2012

21. Falschlehner $\mathrm{C}$ and Boutros $\mathrm{M}$ : Innate immunity: Regulation of caspases by IAP-dependent ubiquitylation. EMBO J 31: 2750-2752, 2012. 